\title{
Bibliography
}

[1] Bourbaki Eléments de mathématique, théorie des ensembles Hermann editor Paris 1963

[2] Mikio Nakahara Geometry, Topology and Physics Graduate student series in Physics Editor Douglas F. Brewer Adam Hilger Bristol and New York Jersey London Hong Kong 1990

[3] John Baez, Javier P. Muniain Gauge Fields, Knots and Gravity World Scientific Singapore-New Jersey-London-Hong Kong 1994

[4] Walter Rubin Real and Complex Analysis McGraw-Hill Book Co., Singapore 1987

[5] see for instance http://mathworld.wolfram.com/VectorSpace.html 
This page intentionally left blank 


\section{List of definitions}

def.1 Manifold. . . . . . . . . . . . . . . . . . . . . . . . . . . . . 1

def.2 Dimension of a covering set of a Manifold. . . . . . . . . . . . . . . . . . . 1

def.3 Differentiable manifold. . . . . . . . . . . . . . . . . . . . . . . . . . 1

def.4 Dimension of a differential manifold. . . . . . . . . . . . . . . . . . . . . 2

def.5 Restriction of a differentiable manifold (definition 3 (p. 1)). . . . . . . . . . . . 3

def.6 Smooth maps on differentiable manifolds. . . . . . . . . . . . . . . . . . . . . 3

def.7 $\mathcal{F}$ is the set of smooth maps from $\mathbf{M}$ to $\mathcal{R}$. . . . . . . . . . . . . . . . . . . . 3

def.8 $\mathcal{F}_{\mathrm{O}} \ldots \ldots \ldots \ldots \ldots$

def.9 Restricted smooth map $\mathcal{F}_{\mathbf{O}}^{\mathbf{M}}$. . . . . . . . . . . . . . . . . . . . . . . . . 3

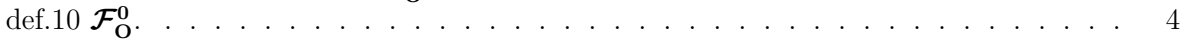

$\operatorname{def} 11 \mathcal{F}_{\mathrm{k}}, \mathcal{F}_{\mathrm{k}}^{\mathrm{M}}, \mathcal{F}_{\mathrm{k}}^{\mathbf{0}} \ldots \ldots \ldots \ldots \ldots \ldots \ldots$

def.12 Constant smooth map. . . . . . . . . . . . . . . . . . . . . . . 5

def.13 ${ }^{\mathrm{i}} \mathrm{g}_{p^{\prime}}(\mathrm{x}, \mathrm{a}),{ }^{\mathrm{i}} \mathrm{g}_{p^{\prime}}^{\prime}(p, \mathrm{a}), \mathrm{g}_{p^{\prime}}(\mathrm{x}, \mathrm{a}), \mathrm{g}_{p^{\prime}}^{\prime}(p, \mathrm{a}) \ldots \ldots \ldots \ldots$

def.14 ${ }^{\mathrm{i}} \mathrm{g}_{p^{\prime}}(\mathrm{x}, \mathrm{a}, \mathrm{b}),{ }^{\mathrm{i}} \mathrm{g}_{p^{\prime}}^{\prime}(p, \mathrm{a}, \mathrm{b}), \mathrm{g}_{p^{\prime}}(\mathrm{x}, \mathrm{a}, \mathrm{b}), \mathrm{g}_{p^{\prime}}^{\prime}(p, \mathrm{a}, \mathrm{b}) \ldots \ldots \ldots$

def.15 Vector fields. . . . . . . . . . . . . . . . . . . . . . 8

def.16 Sum of vector fields. . . . . . . . . . . . . . . . . . . . . . . . . . . 8

def.17 Product of a vector field by a smooth map over a differential manifold. . . . . . 8

def.18 Restriction of a vector field to an open subset of a differentiable manifold. . . . 12

def.19 $\mathbf{v}_{\mathbf{k}}$, the restriction of a vector field $\mathbf{v} \in \mathcal{V}$ to $\mathbf{U}_{\mathbf{k}} \ldots \ldots \ldots$. . . . . . . . . . . 13

def.20 Coordinate maps of $\mathcal{F}_{\mathbf{k}}$. . . . . . . . . . . . . . . . . . . . . . . . . . . . . . 15

def.21 Basic vector field maps $\left(\boldsymbol{\partial}_{\boldsymbol{\mu}}: \mathcal{F}_{\mathrm{k}} \rightarrow \mathcal{F}_{\mathrm{k}}\right) \ldots \ldots \ldots \ldots$

def.22 Finite basis of a set of vector field. . . . . . . . . . . . . . . . . . . . . . . . 17

def.23 $\left(\boldsymbol{\partial}_{\boldsymbol{\mu}}:[\mathbf{1}, \mathbf{n}] \rightarrow \mathcal{V}_{\mathbf{k}}\right)$, canonical basic vectors in the local frame. . . . . . . . . . 17

def.24 Components of a vector field of a local manifold $\mathbf{U}_{\mathbf{k}} \subset \mathbf{M}$. . . . . . . . . . . . 17

def.25 Local components of a vector field. . . . . . . . . . . . . . . . . . . 17

def.26 $\mathbf{u} \sim_{p} \mathbf{v}$ in $\mathcal{V} \ldots \ldots \ldots \ldots$

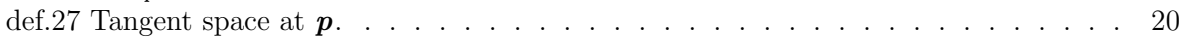

def.28 Operations on tangent space. . . . . . . . . . . . . . . . . . . . . . 20

def.29 Tangent space basis vector ${ }^{[p]} \boldsymbol{\partial}_{\boldsymbol{\mu}}$ of $[p]^{\mathbf{T}} \ldots \ldots \ldots \ldots \ldots \ldots$

def.30 Change of coordinates in a differentiable manifold. . . . . . . . . . . . . . . . 23

def.31 Metric on a differentiable manifold. . . . . . . . . . . . . . . . . . . . . . 25

def.32 Restriction of a metric over an open. . . . . . . . . . . . . . . . . . . . . 25

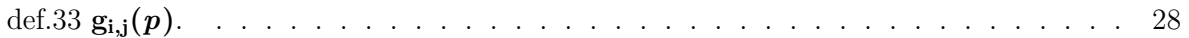

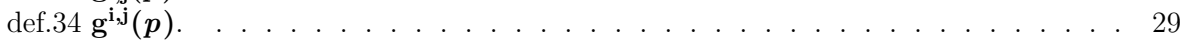

def.35 Signature of a metric. . . . . . . . . . . . . . . . . . . . . 32

def.36 One-form field. . . . . . . . . . . . . . . . . . . . . . . . . 32

def.37 $\mathcal{V}^{*}$, dual of the set of vector fields $\mathcal{V}$ on a differentiable manifold. . . . . . . . . . 32

def.38 $\mathcal{V}_{\mathbf{O}}^{*}$, dual of the set of vector fields $\mathcal{V}_{\mathbf{O}}$ on the restricted manifold $\mathbf{O}$ open subset

of a manifold $\mathbf{M}$ (definition 5 (p. 3)). . . . . . . . . . . . . . . . . 32

def.39 Restricted one-form field to an open. . . . . . . . . . . . . . . . . . . . . . 33

def.40 Differential. . . . . . . . . . . . . . . . . . . . 34

def.41 Restricted differential to an open of M. . . . . . . . . . . . . . . . . . . 34

def.42 Local coordinates of a ${ }_{1 \triangleleft}$ form. . . . . . . . . . . . . . . . . . . . . . . 36

def.43 Metric induced on $\mathcal{V}^{*}$ by the metric $\mathbf{g}$ on $\mathcal{V}$. . . . . . . . . . . . . . . . . . . 40

def.44 Tensorial product of several sets which are either the set linear forms or the set

of vector fields of a differential manifold. . . . . . . . . . . . . . . . . . . . . . . 40

def.45 Tensorial product of a mixed set of vector fields and of linear forms. . . . . . . . 41 
def.46 Tensorial product of several linear forms (versus vector fields). . . . . . . . . . 42

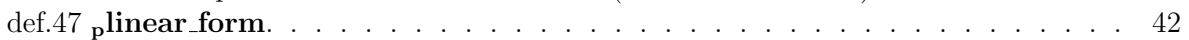

def.48 Metric on a tensorial product. . . . . . . . . . . . . . . . . 45

def.49 Permutation of $\left(\mathcal{V}^{*}\right)^{\mathbf{p}}\left(\operatorname{versus}(\mathcal{V})^{\mathbf{p}}\right) \ldots \ldots \ldots \ldots \ldots$

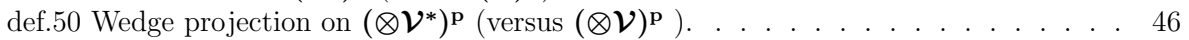

def.51 Wedge product of $\mathbf{p} 1$-linear forms (versus vector fields). . . . . . . . . . . . 47

def.52 p_form. . . . . . . . . . . . . . . . . . . . . . 47

def.53 $\mathcal{I}_{\mathrm{p}} \ldots \ldots \ldots \ldots \ldots \ldots$

def.540 0 form. . . . . . . . . . . . . . . . . . . . . 49

def.55 $\Omega^{0}$. . . . . . . . . . . . . . . . . . . . . . 49

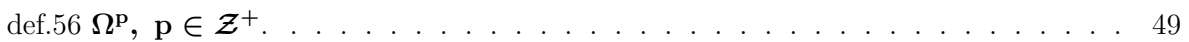

def.57 $\Omega$. . . . . . . . . . . . . . . . . . . . . . . . . 49

def.58 $\mathbf{a} \otimes \mathbf{b}, \mathbf{a} \wedge \mathbf{b}, \mathbf{a} \in \Omega, \mathbf{b} \in \Omega$. . . . . . . . . . . . . . . . . . . . . 49

def.59 The differential operator $\mathbf{d}$ as a map $(\mathbf{d}: \boldsymbol{\Omega} \rightarrow \boldsymbol{\Omega})$. . . . . . . . . . . . . . . 52

def.60 Oriented volume form. . . . . . . . . . . . . . . . . . . . . 54

def.61 Volume form attached to a metric. . . . . . . . . . . . . . . . . . . . 58

def.62 Hodge $*$ operator. . . . . . . . . . . . . . . . . . . . . . . . . . . . . . . . 59

def.63 Oriented Manifold. . . . . . . . . . . . . . . . . . . . . . 61

def.64 Two local bases with the same orientation. . . . . . . . . . . . . . . . . 61

def.65 Oriented integral of an element of $\mathcal{F}$. . . . . . . . . . . . . . . . . . . . . 65

def.66 Oriented integral of an element of $\boldsymbol{\Omega}^{\mathbf{n}}$ (see definition 56 (p. 49)) . . . . . . . . 65

def.67 Oriented surface integral. . . . . . . . . . . . . . . . . . . . . . . 65

def.68 Smooth surface. . . . . . . . . . . . . . . . . . . . . 65

def.69 Integral of an $\mathbf{n}-\mathbf{1}$ differential form on a smooth surface. . . . . . . . . . . . 66

def.70 Flux of a linear ${ }_{1}$ form through a smooth surface (definition 68 (p. 65)) which is

the boundary (definition 138 (p. 157)) of a convex differentiable manifold (definition

71 (p.67)). . . . . . . . . . . . . . . . . . . 67

def.71 Convex differentiable manifold of dimension n. . . . . . . . . . . . . . . . . . 67

def.72 Convex smooth surface of dimension $\mathbf{n}-\mathbf{1}$. . . . . . . . . . . . . . . . . 70

def.73 Interior of a convex smooth surface. . . . . . . . . . . . . . . . . . . . 71

def.74 Lie bracket of two vector fields. . . . . . . . . . . . . . . . . . . . . . . 85

def.75 Bundle. . . . . . . . . . . . . . . . . . . . . . 86

def.76 Fibers of a bundle. . . . . . . . . . . . . . . . . . . . . 86

def.77 Vector bundle. . . . . . . . . . . . . . . . . . . . . . . . . 86

def.78 Sections of a vector bundle (definition 77 (p. 87)), section module. . . . . . . . 87

def.79 Basis of the section module. . . . . . . . . . . . . . . . . . . . . . 87

def.80 Dual of the section module. . . . . . . . . . . . . . . . . . . . . . 88

def.81 Dual of a basis of the section module. . . . . . . . . . . . . . . . . . . . . 88

def.82 Standard basis of the section module and of its dual. . . . . . . . . . . . . . . . 88

def.83 Linear map or linear transformation on the sections of a vector bundle (definition

77 (p. 87)). . . . . . . . . . . . . . . . . . . . . 89

def.84 End_E. . . . . . . . . . . . . . . . . . . . . . . . . . . . . . . . . . . . . . 89

def.85 Multilinear map or ${ }_{\mathbf{p}}$ linear_form on a bundle. . . . . . . . . . . . . . . . . . 90

def.86 Covariant derivative on a vector bundle (definition 77 (p. 87)) . . . . . . . . . . 90

def.87 Connection on a bundle. . . . . . . . . . . . . . . . . . . . . . . 90

def.88 $\mathbf{D}^{0}$. . . . . . . . . . . . . . . . . . . . . . . . . . . 91

def.89 Vector potential. . . . . . . . . . . . . . . . . . . . . . . 91

def.90 Connection $\mathbf{D}^{\text {End_E }}$ induced by a connection $\mathbf{D}$ on a vector bundle $\mathbf{E}$. . . . . . . 92

def.91 Derivative and differential of linear transformations on a bundle. . . . . . . . . 93 
def.92 Covariant derivative on the dual of a bundle. . . . . . . . . . . . . . . . . . . 94

def.93 Covariant derivative on multilinear forms on a bundle. . . . . . . . . . . . . . 95

def.94 Covariant derivative on tensorial product of bundles. . . . . . . . . . . . . . . 95

def.95 Covariant derivative of a metric. . . . . . . . . . . . . . . . . . . . 96

def.96 Differential of a section associated to a connection. . . . . . . . . . . . . . . 96

def.97 Wedge product of a term of $\mathbf{V}_{\mathbf{s}} \otimes \boldsymbol{\Omega}^{\mathbf{p}}$ and a term of $\mathbf{V}_{\mathbf{s}} \otimes \boldsymbol{\Omega}^{\mathbf{p}^{\prime}}$. . . . . . . . . . 97

def.98 Definition of $\left(\mathbf{d}_{\mathbf{D}} \mathbf{t}: \mathbf{V}_{\mathbf{s}} \otimes \Omega^{\mathbf{p}} \rightarrow \mathbf{V}_{\mathbf{s}} \otimes \Omega^{\mathbf{p}+\mathbf{1}}\right)$, a map of $\mathbf{V}_{\mathbf{s}} \otimes \Omega^{\mathbf{p}}$ to $\mathbf{V}_{\mathbf{s}} \otimes \Omega^{\mathbf{p}+\mathbf{1}}$

(See section 1.11 (p. 49)) . . . . . . . . . . . . . . . . . . . . . . . . . 97

def.99 Parallel transport. . . . . . . . . . . . . . . . . . . . . . . . . . . . 98

def.100 Curvature. . . . . . . . . . . . . . . . . . . . . . . . . 98

def.101 Metric in general relativity. . . . . . . . . . . . . . . . . . . . 108

def.102 Tangent space bundle, vector bundle (definition 77 (p. 87)) in general relativity

in dimension $\mathbf{n} . \quad$. . . . . . . . . . . . . . . . . . . . . . . . . . . . . . . . . . . . . . . . . . 109

def.103 Section attached to a vector field. . . . . . . . . . . . . . . . . 109

def.104 Levi-Civita connection $\boldsymbol{\nabla}$. . . . . . . . . . . . . . . . . . . . . . . . . . 109

def.105 Christoffel symbols. . . . . . . . . . . . . . . . . . . . 110

def.106 Riemann curvature. . . . . . . . . . . . . . . . . . . . . . 112

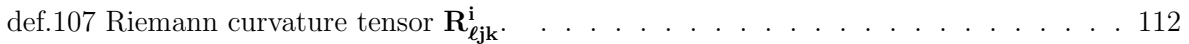

def.108 Ricci tensor. . . . . . . . . . . . . . . . . . . . . . . . . . . . 113

def.109 Scalar curvature. . . . . . . . . . . . . . . . . . . . 113

def.110 Einstein Hilbert Lagrangian. . . . . . . . . . . . . . . . . . . . . . 117

def.111 Einstein tensor. . . . . . . . . . . . . . . . . . . . . . . . . . . . 122

def.112 Map. . . . . . . . . . . . . . . . . . . . . . . . . . 131

def.113 Identity map. . . . . . . . . . . . . . . . . . . . . . . . . . . . 132

def.114 Image of a subset given by a map. . . . . . . . . . . . . . . . . . . . . . . . 132

def.115 Inverse image of a subset given by a map. . . . . . . . . . . . . . . . . . . . 133

def.116 Inverse image of an element given by a map. . . . . . . . . . . . . . . . . . 134

def.117 Product of maps. . . . . . . . . . . . . . . . . . . . . . . . . . . . . 134

def.118 Restriction of a map to a subset. . . . . . . . . . . . . . . . . . . . 135

def.119 Product of sets. . . . . . . . . . . . . . . . . . . . . 135

def.120 Product of maps induced by product of sets. . . . . . . . . . . . . . . . 135

def.121 Projection on product of sets. . . . . . . . . . . . . . . . . . . . . 136

def.122 Injective map, one-to-one map. . . . . . . . . . . . . . . . . . . . . . . . . 139

def.123 Surjective map, onto map. . . . . . . . . . . . . . . . . . . . . . . . . . . . 139

def.124 Bijective map or bijection. . . . . . . . . . . . . . . . . . . . . . . . . . . 139

def.125 Inverse map of a map. . . . . . . . . . . . . . . . . . . . . . . . . . . . . 140

$\operatorname{def} 126\left(\mathrm{x}^{1}, \mathrm{x}^{2}, \ldots, \mathrm{x}^{\mathrm{n}}\right),\left(\mathrm{x}_{1}, \mathrm{x}_{2}, \ldots, \mathrm{x}_{\mathrm{n}}\right), \prod_{\mathrm{i} \in \mathrm{I}}^{\mathrm{e}} \mathrm{x}^{\mathrm{i}} \ldots \ldots \ldots \ldots \ldots \ldots$

$\operatorname{def} 127 \sum \mathrm{x}^{\mathrm{i}}, \mathbf{n} \in \mathcal{Z}^{+} \ldots \ldots \ldots \ldots \ldots \ldots \ldots \ldots$

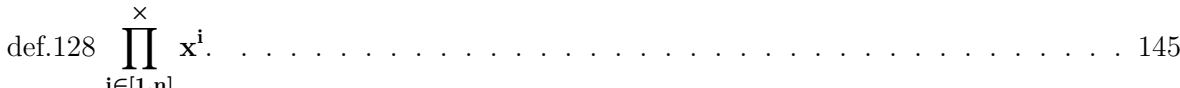

def.129 Restriction of a map defined on a product of sets. . . . . . . . . . . . . . . 145

def.130 Transformed of a product of sets by a map. . . . . . . . . . . . . . . . . . . 145

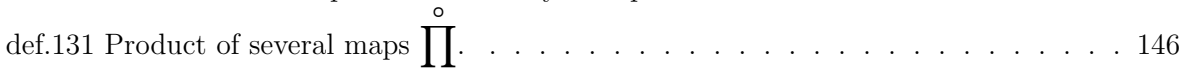

def.132 Open subset. . . . . . . . . . . . . . . . . . . . . 151

def.133 Induced topology on subset. . . . . . . . . . . . . . . . . . . . 151 
def.134 Induced topology on product of spaces (definition 119 (p. 135)) . . . . . . . . . 153

def.135 Closed subset. . . . . . . . . . . . . . . . . . . . . 154

def.136 Closure of a subset. . . . . . . . . . . . . . . . . . . 155

def.137 Interior of a subset. . . . . . . . . . . . . . . . . . . . . . 156

def.138 Boundary of a subset. . . . . . . . . . . . . . . . . . . . 157

def.139 Separated topology (Hausdorff space). . . . . . . . . . . . . . . . . . . 159

def.140 Compact set. . . . . . . . . . . . . . . . . . . . . . . . . . . . . . . . . 159

def.141 Continuous map. . . . . . . . . . . . . . . . . . . . . . . . 165

def.142 Isomorphism. . . . . . . . . . . . . . . . . . . . . . . . 166

def.143 Real numbers, $\mathcal{R}$. . . . . . . . . . . . . . . . . . . . . 170

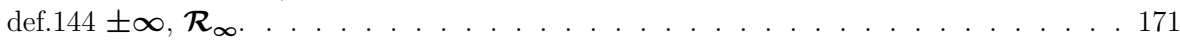

def.145 Order on $\mathcal{R}_{\infty}$. . . . . . . . . . . . . . . . . . . . . . . . . 171

$\operatorname{def} 146 \min _{\mathbf{i} \in \mathrm{I}} \mathbf{a}(\mathbf{i}), \max _{\mathbf{i} \in \mathrm{I}} \mathbf{a}(\mathbf{i}) \ldots \ldots \ldots \ldots$

def.147 Euclidean Metric, Euclidean Metric space. . . . . . . . . . . . . . . . . . . 176

def.148 Minimum distance of a point to a subset of a metric set. . . . . . . . . . . 176

def.149 Induced Euclidean Metric. . . . . . . . . . . . . . . . . . . . . 176

def.150 Open ball on a Euclidean metric space. . . . . . . . . . . . . . . . . . . . 176

def.151 Open subsets on a Euclidean metric set. . . . . . . . . . . . . . . . . . . . 177

def.152 Closed ball on a Euclidean metric space. . . . . . . . . . . . . . . . . . . . . 179

def.153 Sign of a real number. . . . . . . . . . . . . . . . . . . . . . . . . . . 181

def.154 Absolute value of a real number. . . . . . . . . . . . . . . . . . . . . . 181

def.155 Open interval (see definition 378 (p. 484)) . . . . . . . . . . . . . . . . . 181

def.156 Closed interval. . . . . . . . . . . . . . . . . . . . . 181

def.157 Limit of a map at a point. . . . . . . . . . . . . . . . . . . . . . . . . 182

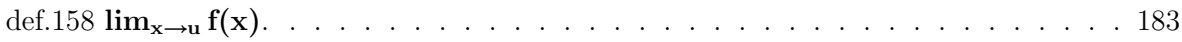

def.159 Continuous map at a point. . . . . . . . . . . . . . . . . . . . . . . 183

def.160 Uniformly continuous map defined between a subset $\mathbf{C}$ of $\mathbf{A}$ and a set $\mathbf{B}$, the

topology being induced by a Euclidean metric. . . . . . . . . . . . . . . . . . . 184

def.161 Derivative of a function at a point. . . . . . . . . . . . . . . . . 192

def.162 Derivable function. . . . . . . . . . . . . . . . . . . . . 192

def.163 $\frac{\mathrm{d}^{\mathrm{p}}}{\mathrm{dx}^{\mathrm{p}}}$. . . . . . . . . . . . . . . . . . . . . . . . . . . . 193

def.164 Notations for the derivative of a derivable function. . . . . . . . . . . . . . 193

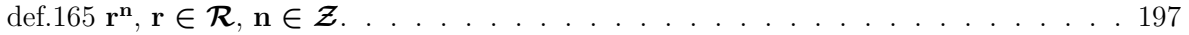

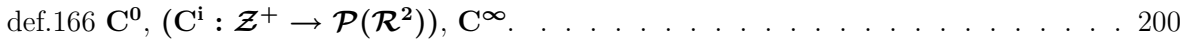

def.167 Group. . . . . . . . . . . . . . . . . . . . . . 205

def.168 Subgroup. . . . . . . . . . . . . . . . . . . . 206

def.169 Map conserving the internal law. . . . . . . . . . . . . . . . . . . 206

def.170 Commutative group or Abelian group. . . . . . . . . . . . . . . . . 206

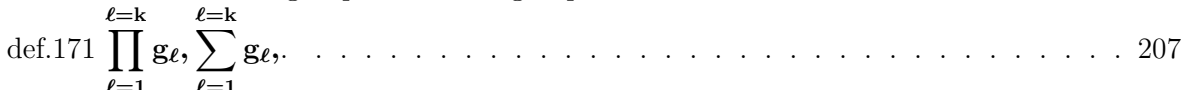

def.172 Permutation of $[\mathbf{1}, \mathbf{n}] . \ldots \ldots$. . . . . . . . . . . . . . . . . 207

def.173 Transposition. . . . . . . . . . . . . . . . . . . . . . . . . 208

def.174 Transposition of two consecutive terms. . . . . . . . . . . . . . . . 208

def.175 Signature of a permutation. . . . . . . . . . . . . . . . . . . . . . . . . . . . . . . . . . . . . . . . . .

def.176 Permutation with identical elements. . . . . . . . . . . . . . . . . 210

def.177 Product $\prod$, Sum $\sum$ of a finite ordered subset of a group. . . . . . . . . . . . 211

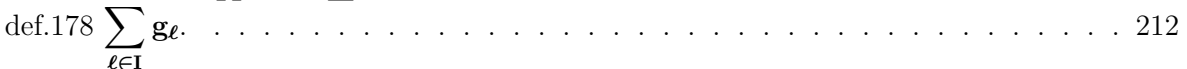


def.179 Ring F. . . . . . . . . . . . . . . . . . . . . . . . 214

def.180 Commutative ring F. . . . . . . . . . . . . . . . . . . . . 215

def.181 Field. . . . . . . . . . . . . . . . . . . . . . . . . . 217

def.182 Module over a commutative ring F . . . . . . . . . . . . . . . . . . . . . . 218

def.183 Submodule of a module. . . . . . . . . . . . . . . . . . . . . . . . . . . . 219

def.184 Finite basis in a module over a commutative ring F . . . . . . . . . . . . . . . 219

def.185 Independent finite basis in a module over a commutative ring. . . . . . . . . . 219

def.186 Linear maps, linear forms, linear transformations. . . . . . . . . . . . . . . . 220

def.187 Dual of a module over a commutative ring. . . . . . . . . . . . . . . . . . 221

def.188 Dual of a module over another module both built on a commutative ring. . . . 221

def.189 Induced dual basis. . . . . . . . . . . . . . . . . . . . . . . . . 222

def.190 Induced basis of the dual of a module over another module. . . . . . . . . . . 223

def.191 Multilinear maps or Multilinear form. . . . . . . . . . . . . . . . . . . . . 223

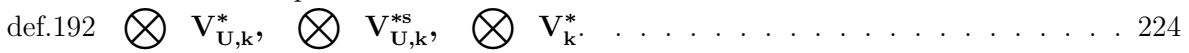

$\mathrm{k} \in[1, \mathrm{~K}] \quad \mathrm{k} \in[1, \mathrm{~K}] \quad \mathrm{k} \in[1, \mathrm{~K}]$

def.193 Operations on multilinear maps. . . . . . . . . . . . . . . . . . . . . . . . . 224

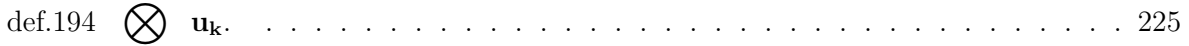

$\mathrm{k} \in[1, \mathrm{~K}]$

def.195 Tensorial product. . . . . . . . . . . . . . . . . . . . . . 230

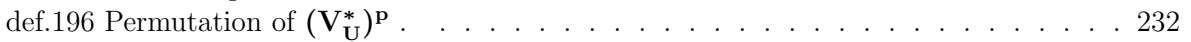

def.197 Wedge projection on $\left(\otimes \mathbf{V}_{\mathrm{U}}^{*}\right)^{\mathbf{p}} \ldots \ldots . \ldots . \ldots . \ldots . \ldots . \ldots 233$

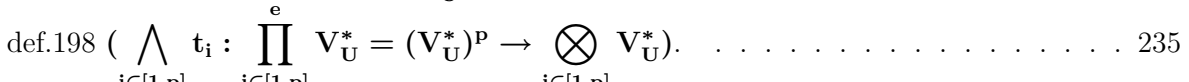

def.199 Vector space. . . . . . . . . . . . . . . . . . . . . . . 235

def.200 $\sum_{\ell \in \mathrm{I}} \mathbf{v}_{\ell} \ldots \ldots \ldots \ldots \ldots \ldots \ldots$

def.201 Sub-vector space. . . . . . . . . . . . . . . . . . . . . . . . . 237

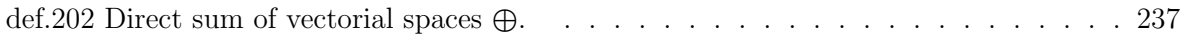

def.203 Norm on a vector space. . . . . . . . . . . . . . . . . . . . . . . . 237

def.204 Linear combination of vectors. . . . . . . . . . . . . . . . . . . . . . . . 239

def.205 Bases of a vectorial space. . . . . . . . . . . . . . . . . . . . . . . 239

def.206 Independent vectors set. . . . . . . . . . . . . . . . . . . . . . . . . . . . 239

def.207 Vector space with finite dimension. . . . . . . . . . . . . . . . . . . . . . . . 240

def.208 Dimension of a vectorial space with finite dimension. . . . . . . . . . . . . . . 240

def.209 Dimension of a subset of a vectorial space with finite dimension. . . . . . . . . 240

def.210 Independent bases of a finite vector space. . . . . . . . . . . . . . . . . . . . 241

def.211 Line and plane in a vectorial space. . . . . . . . . . . . . . . . . . . . . . . 242

def.212 Linear form or linear map on a vectorial space. . . . . . . . . . . . . . . . . . 244

def.213 Dual space. . . . . . . . . . . . . . . . . . . . . . . . . . 244

def.214 Bases $\mathbf{e}^{\mathbf{j}}:[\mathbf{1}, \mathbf{n}] \rightarrow \mathbf{V}^{*}$. . . . . . . . . . . . . . . . . . . . 245

def.215 Kronecker symbol $\delta_{\mathrm{j}}^{\mathrm{i}}=\boldsymbol{\delta}_{\mathrm{ij}}=\boldsymbol{\delta}^{\mathrm{ij}}$. . . . . . . . . . . . . . . . . 245

def.216 Heaviside map $\mathcal{Y}(\mathbf{x})$. . . . . . . . . . . . . . . . . . . . . . . . . . . 245

def.217 Multilinear forms on a vectorial space. . . . . . . . . . . . . . . . . . 246

def.218 $\left(\otimes \mathbf{V}^{*}\right)^{\mathbf{p}}$. . . . . . . . . . . . . . . . . . . . . . . . . . . 246

$\operatorname{def} .219\left(\bigotimes_{\mathbf{k} \in[1, \mathbf{p}]} \mathbf{u}_{\mathbf{k}}: \prod_{\mathbf{k} \in[1, \mathbf{p}]}\left(\mathbf{V}^{*}\right) \rightarrow \bigotimes_{\mathbf{k} \in[1, \mathbf{p}]} \mathbf{V}^{*}=\left(\otimes \mathbf{V}^{*}\right)^{\mathbf{p}}\right)$. . . . . . . . . . . 246

def.220 Wedge projection on $\left(\otimes \mathbf{V}^{*}\right)^{\mathbf{p}}$. . . . . . . . . . . . . . . . . . . . . . . . 247

def.221 Determinant. . . . . . . . . . . . . . . . . . . . . . 249

def.222 Tensorial product. . . . . . . . . . . . . . . . . . . . . . 250

def.223 Basis of a p_tensorial product of a finite dimension vector space. . . . . . . . 250 
def.224 Linear transformation. . . . . . . . . . . . . . . . . . . . . . . 250

def.225 Matrix of a linear transformation. . . . . . . . . . . . . . . . . 250

def.226 Transposition of a matrix. . . . . . . . . . . . . . . . . . . . . . . 252

def.227 Determinant of a matrix. . . . . . . . . . . . . . . . . . . 252

def.228 Minor of a term of a matrix. . . . . . . . . . . . . . . . . . . . . . . 254

def.229 Determinant of a linear transformation of a vectorial space of finite dimension

on itself. . . . . . . . . . . . . . . . . . . . . . . . . . 256

def.230 Linear equations. . . . . . . . . . . . . . . . . . . . . . . 258

def.231 RC . . . . . . . . . . . . . . . . . . . . . . . . 259

def.232 Norm and complex conjugate on $\boldsymbol{R} \mathcal{C}$. . . . . . . . . . . . . . . . . . . . . . . 259

def.233 Norm and complex conjugate on $\mathcal{R}$. . . . . . . . . . . . . . . . . . . . 260

def.234 Inner product (Riemannian inner product), adjoint of a vector. . . . . . . . . . 261

def.235 Matrix associated to an inner product. . . . . . . . . . . . . . . . 262

def.236 Inner product when $\mathcal{R C}=\boldsymbol{R}$. . . . . . . . . . . . . . . . . . . . . . . . 262

def.237 Euclidean vector space, Euclidean norm. . . . . . . . . . . . . . . . . . 263

def.238 Orthogonal vectors. . . . . . . . . . . . . . . . . . . . 263

def.239 Adjoint transformation over an inner product. . . . . . . . . . . . . . . . 264

def.240 Unitary transformation. . . . . . . . . . . . . . . . . . . . 265

def.241 $\mathcal{R}^{\mathrm{n}}$. . . . . . . . . . . . . . . . . . . . . . . 266

def.242 Reference or standard basis of $\boldsymbol{R}^{\mathbf{n}}$. . . . . . . . . . . . . . . . . . . . . . . . 267

def.243 p_norm in $\mathcal{R}^{\mathbf{n}}$. . . . . . . . . . . . . . . . . . . . . . . . . . 267

def.244 Projection of $\boldsymbol{R}^{\mathbf{n}}$ on $\boldsymbol{R}^{\mathbf{n}-1}$. . . . . . . . . . . . . . . . . . . . . . . 270

def.245 Complex numbers $\mathcal{C}$. . . . . . . . . . . . . . . . . . . . . . 271

def.246 Multiplication and addition on complex numbers. . . . . . . . . . . . . . 272

def.247 Norm of a complex number. . . . . . . . . . . . . . . . . . . . . . 272

def.248 Complex conjugate of a complex number. . . . . . . . . . . . . . . . . . 272

def.249 Semi Riemannian inner product (or Semi Riemannian metric). . . . . . . . . . 273

def.250 Set of orthogonal vectors. . . . . . . . . . . . . . . . . . . . . . 274

def.251 Length of a vector. . . . . . . . . . . . . . . . . . . . . . . . . . 274

def.252 Signature of a vectorial space with a semi Riemannian inner product (metric). . 276

def.253 Adjoint transformation over a semi Riemannian inner product. . . . . . . . . 276

def.254 Unitary transformation within a semi Riemannian inner product. . . . . . . . 277

def.255 Convex subset. . . . . . . . . . . . . . . . . . . . . 277

def.256 Dimension of a convex subset. . . . . . . . . . . . . . . . . . . . . 280

def.257 The inner part of a finite set of vectors of a vectorial space. . . . . . . . . . . 281

$\operatorname{def} 258 \sum_{\mathrm{i} \in \emptyset} \mathbf{i}=\mathbf{0}, \sum_{\mathrm{i} \in \emptyset} \mathbf{v}(\mathbf{i})=\mathbf{0} \ldots \ldots \ldots \ldots . \ldots \ldots$

def.259 The inner part of a box defined by a basis. . . . . . . . . . . . . . . . . . 281

def.260 Open ball (and open cube) of $\boldsymbol{R}^{\mathbf{n}}$. . . . . . . . . . . . . . . . . . . . . 285

def.261 Open cube. . . . . . . . . . . . . . . . . . . . . . 285

def.262 Closed cube. . . . . . . . . . . . . . . . . . . . . . . . 285

def.263 Open of the $\boldsymbol{R}^{\mathrm{n}}$ topology. . . . . . . . . . . . . . . . . . . . . . . . . . . 286

def.264 Projection of $\mathcal{R}^{\mathbf{n}}$ on $\mathcal{R}^{\mathbf{n}-\mathbf{1}}$ along the axis i: $\left(\operatorname{Pr}_{\mathbf{i}}: \mathcal{R}^{\mathbf{n}} \rightarrow \mathcal{R}^{\mathbf{n}-\mathbf{1}}\right)$. . . . . . 289

def.265 Functions on $\boldsymbol{R}^{\mathrm{n}}$. . . . . . . . . . . . . . . . . . . . . . . . . . . 296

def.266 Sequence. . . . . . . . . . . . . . . . . . . . . . . . . 297

def.267 Accumulation point of a sequence on a topological space. . . . . . . . . . . . 297

def.268 Limit of a sequence. . . . . . . . . . . . . . . . . . . . . . . . . . . . . . 298

def.269 Cauchy sequence. . . . . . . . . . . . . . . . . . . . . . . 300

def.270 Accumulation point and limit for a sequence in $\boldsymbol{\mathcal { R }}_{\infty}$. . . . . . . . . . . . . . 301

def.271 Sequence of maps. . . . . . . . . . . . . . . . . . . . . . . . . . 302 
def.272 Sequence of maps converging uniformly to a map limit on a set. . . . . . . . . 302

def.273 Cauchy sequence of maps. . . . . . . . . . . . . . . . . . . . . . . . 302

def.274 Uniform Cauchy sequence of maps. . . . . . . . . . . . . . . . . . . . . . 303

def.275 Partial derivative of a map, $\partial_{\mathbf{i}}=\frac{\partial}{\partial x^{\mathbf{i}}}$. . . . . . . . . . . . . . . . . . . 304

def.276 Derivative operator, product of derivative operators, $\mathbf{C}^{\mathbf{i}}, \mathbf{D}^{\mathbf{k}}$. . . . . . . . . 304

def.277 $\mathbf{C}^{\infty}$. . . . . . . . . . . . . . . . . . . . . . 304

def.278 Smooth map, map infinitely derivable, $\mathrm{C}^{\infty}\left\{\mathcal{R}^{\mathrm{m}} \rightarrow \mathcal{R}^{\mathrm{n}}\right\}$, map with partial

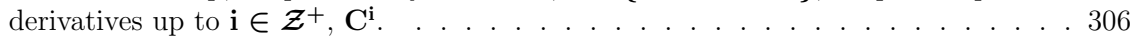

def.279 Boxes in $\boldsymbol{R}^{\mathbf{n}}$. . . . . . . . . . . . . . . . . . . . . . . . . 320

def.280 Hybrid box. . . . . . . . . . . . . . . . . . . . . 320

def.281 Corners or summits of a box. . . . . . . . . . . . . . . . . . . . . . 322

def.282 Ridge of a box. . . . . . . . . . . . . . . . . . . . . . . . . . . . . . . . . . . . . . . . . . . . . .

def.283 Face of a box. . . . . . . . . . . . . . . . . . . . . . . . . . . . . . 324

def.284 Path connection relation. . . . . . . . . . . . . . . . . . . . 326

def.285 Path connected subset. . . . . . . . . . . . . . . . . . . . . . . . . 327

def.286 Path connected part. . . . . . . . . . . . . . . . . . . . . . 327

def.287 Map $\left(\mathbf{f}: \boldsymbol{\mathcal { R }}^{\mathbf{n}} \rightarrow \boldsymbol{\mathcal { R }}^{\mathrm{m}}\right)$ with compact support. . . . . . . . . . . . . . . . . 331

def.288 Set of integration grids: $\mathbf{G r}$ and its associated sum map $\left(\mathrm{gr}^{\mathrm{f}}:\left(\mathcal{R}^{\mathrm{m}}\right)^{\mathcal{R}^{\mathrm{n}}} \times \mathbf{G r} \rightarrow\right.$

$\boldsymbol{R}^{\mathbf{m}}$ ) (see definition 373 (p. 474)). . . . . . . . . . . . . . . . . . . . . . . . . . 331

def.289 Set of grids with a mesh size smaller than a positive real number: $\mathbf{G r}_{\mathbf{a}}$, a $\in \mathcal{R}^{+}$. 332

def.290 Riemann integral $\mathbf{J}(\mathbf{f})$ of a map $\left(\mathbf{f}: \boldsymbol{\mathcal { R }}^{\mathbf{n}} \rightarrow \boldsymbol{\mathcal { R }}^{\mathbf{m}}\right)$ with a compact support. . . 332

def.291 Translated grid. . . . . . . . . . . . . . . . . . . . . 334

def.292 Set of Riemann integrals with a compact support converging uniformly. . . . . 337

def.293 Characteristic map of a subset of $\boldsymbol{R}^{\mathbf{n}}$. . . . . . . . . . . . . . . . . . . . . . 341

def.294 Integration over a set. . . . . . . . . . . . . . . . . . . . . . 341

def.295 Volume of a subset $\mathbf{A} \subset \mathcal{R}^{\mathbf{n}}$ which is within a cube of $\boldsymbol{R}^{\mathbf{n}} \ldots \ldots . . . . .342$

def.296 $\left(\phi^{\mathbf{1}}(\mathbf{w}): \mathcal{R} \rightarrow\{\mathbf{0}, \mathbf{1}\}\right) \ldots \ldots . \ldots \ldots 1$

def.297 Jacobian of a coordinate change. . . . . . . . . . . . . . . . . . . . . . 358

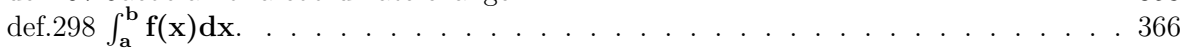

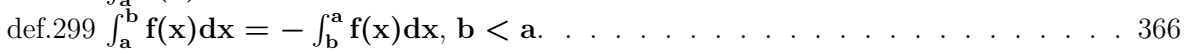

def.300 Differential equations. . . . . . . . . . . . . . . . . . . . . . . . . . 369

def.301 $\mathbf{x}_{\mathbf{p}}$ sequence. . . . . . . . . . . . . . . . . . . . . . . . 369

def.302 $\sigma$-algebra. . . . . . . . . . . . . . . . . . . . . . . . . . . . . . . . 377

def.303 Borel $\boldsymbol{\sigma}$-algebra. . . . . . . . . . . . . . . . . . . . . . . . . . . . . . . . 378

def.304 Measurable space. . . . . . . . . . . . . . . . . . . . . . . . . . 381

def.305 Measure. . . . . . . . . . . . . . . . . . . . . . . . . . 381

def.306 Lebesgue measure of a compact set of $\boldsymbol{\mathcal { R }}^{\mathrm{n}}$. . . . . . . . . . . . . . . . . . . . 382

def.307 Lebesgue integral of a positive continuous map over a compact set of $\boldsymbol{\mathcal { R }}^{\mathbf{n}}$. . . . 382

def.308 Lebesgue measure of an open of $\boldsymbol{R}^{\mathbf{n}}$. . . . . . . . . . . . . . . . . . . . . . 383

def.309 Lebesgue integral of a positive continuous map over an open of $\boldsymbol{\mathcal { R }}^{\mathbf{n}}$. . . . . . . 383

def.310 Lebesgue measure of an element of the Borel $\boldsymbol{\sigma}$-algebra of $\boldsymbol{\mathcal { R }}^{\mathbf{n}}$ (definition 303

(p. 378)).

391

def.311 Lebesgue integral of a positive continuous map over an an element of the Borel

$\sigma$-algebra of $\mathcal{R}^{\mathrm{n}}$.

def.312 Product of a finite set of partial derivatives. . . . . . . . . . . . . . . . 398

def.313 Complex derivative (see section 2.13 (p. 271)) . . . . . . . . . . . . . . . . . 403

def.314 Holomorphic map. . . . . . . . . . . . . . . . . . . . . . . . . . . . 403

def.315 Vectorial space $\mathbf{E}$ needed for the exponentials. . . . . . . . . . . . . . . . 405

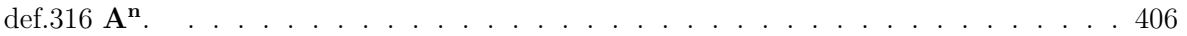


def.317 Exponential. . . . . . . . . . . . . . . . . . . . . . . 407

def.318 sine and cosine. . . . . . . . . . . . . . . . . . . 413

def.319 $\pi$. . . . . . . . . . . . . . . . . . . . . . 413

def.320 Polynomial. . . . . . . . . . . . . . . . . . . . . . . 416

def.321 Polynomial expression. . . . . . . . . . . . . . . . . . . . . . . 416

def.322 Roots of a polynomial, multiplicity of a root. . . . . . . . . . . . . . . . 421

def.323 Eigenvectors, eigenvalues. . . . . . . . . . . . . . . . . . . . . . 428

def.324 A self adjoint linear transformation. . . . . . . . . . . . . . . . . . . . . . . 429

def.325 Terms. . . . . . . . . . . . . . . . . . . . . 433

def.326 Relations. . . . . . . . . . . . . . . . . . . . . 433

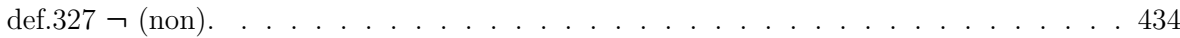

def.328 $\vee$ (or) . . . . . . . . . . . . . . . . . . . . . 434

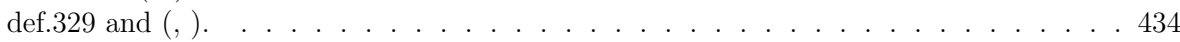

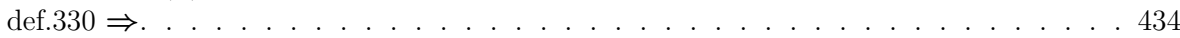

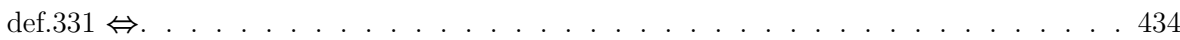

def.332 $\tau_{\mathrm{x}} \mathrm{R}$ or $\tau_{\mathrm{x}}(\mathrm{R}(\mathrm{x}))$. . . . . . . . . . . . . . . . . . . . . 440

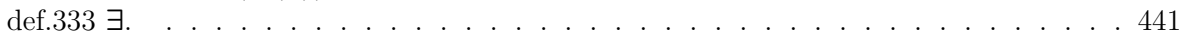

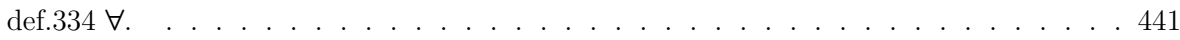

def.335 Functional relations. . . . . . . . . . . . . . . . . . . . . 445

def.336 Equivalence relation. . . . . . . . . . . . . . . . . . . . . . . . . 445

def.337 Representative of an equivalence relation. . . . . . . . . . . . . . . . . 446

def.338 Ordering relation. . . . . . . . . . . . . . . . . . . . 446

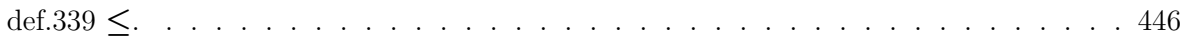

def.340<. . . . . . . . . . . . . . . . . . . . . . . 446

def.341 Opposite order. . . . . . . . . . . . . . . . . . . . . . . . . . . . . . . . . . . . . . . . . . . . . . . . . . .

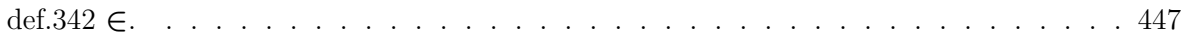

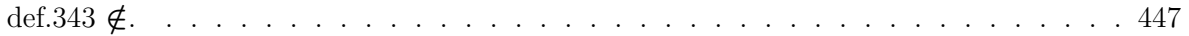

def.344 $\operatorname{coll}_{\mathrm{x}} \mathrm{R}(\mathrm{x})$ and $\operatorname{set}_{\mathrm{x}}\{\mathrm{R}(\mathrm{x})\} \ldots \ldots \ldots 47$

def.345 Set of one or two elements. . . . . . . . . . . . . . . . . . . . . . 447

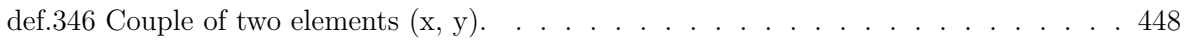

def.347 Projection $\mathbf{p r o j}_{1}, \mathbf{p r o j}_{2}$ of a couple z. . . . . . . . . . . . . . . . . . . 448

def.348 Graph. . . . . . . . . . . . . . . . . . . . . . . . . . . . . 449

def.349 A $\subset$ B. . . . . . . . . . . . . . . . . . . . . . 451

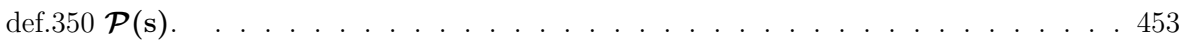

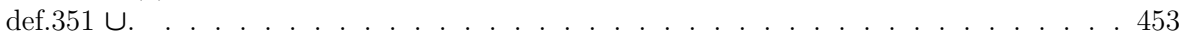

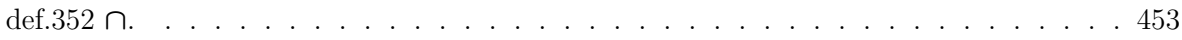

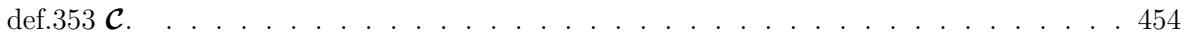

def.354 Subtractive notation for $\mathcal{C}: \mathbf{A}-$ B . . . . . . . . . . . . . . . . . . 454

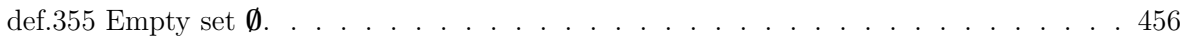

def.356 Product of two sets (see also proposition 822 (p. 473)) . . . . . . . . . . . . . 458

def.357 Map, graph of a map. . . . . . . . . . . . . . . . . . . . 460

def.358 f(x) . . . . . . . . . . . . . . . . . . . . . . . 460

def.359 Injective map, one-to-one map. . . . . . . . . . . . . . . . . . . . . . . . . 461

def.360 Surjective map, onto map. . . . . . . . . . . . . . . . . . . . . . . . . . 461

def.361 Bijective map or bijection. . . . . . . . . . . . . . . . . . 461

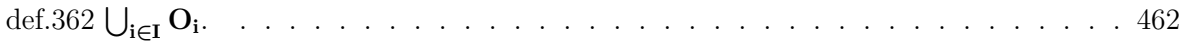

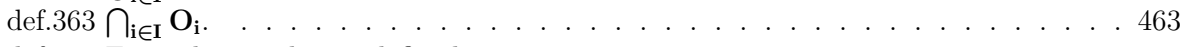

def.364 Equivalence relation defined on a set. . . . . . . . . . . . . . . . . 468 
def.365 Equivalence classes on a set associated to an equivalence relation and to an element of the set.

def.366 Set of equivalence classes on a set associated to an equivalence relation. . . . . 469

def.367 Representative of an equivalence class.

def.368 Set of representatives of the equivalence class of an equivalence relation defined on a set.

def.369 Disjoint set of sets. . . . . . . . . . . . . . . . . . . . . 470

def.370 Partition of a set. . . . . . . . . . . . . . . . . . . 470

def.371 $\sim$ relation between two sets: two sets have the same number of elements. . . . 471

def.372 Product of sets over a set of indices. . . . . . . . . . . . . . . . . 473

def.373 Exponentiation of a set $\mathbf{A}$ by a set $\mathbf{B}: \mathbf{A}^{\mathbf{B}}$. . . . . . . . . . . . . . . . 474

def.374 Ordered sets. . . . . . . . . . . . . . . . . . . . . 482

def.375 Induced Order on a subset. . . . . . . . . . . . . . . . . . . . 483

def.376 Graph of an order on a set. . . . . . . . . . . . . . . . . . . . . . . . 483

def.377 Total order on a set. . . . . . . . . . . . . . . . . . . . . . 483

def.378 Intervals. . . . . . . . . . . . . . . . . . . . . . . . . . 484

def.379 Smallest (resp. biggest) element of a set. . . . . . . . . . . . . . . . . . . . 484

def.380 Minoring (resp. Majoring) element. . . . . . . . . . . . . . . . . . . 484

def.381 upper_limit (resp. lower_limit) of a subset. . . . . . . . . . . . . . . . . 485

def.382 max or min of a subset. . . . . . . . . . . . . . . . . . . . . . . . . . 485

def.383 maximal (resp. minimal) of an ordered set. . . . . . . . . . . . . . . . . 485

def.384 Increasing map. . . . . . . . . . . . . . . . . . . . . . . . . . . . . . . . . 485

def.385 Decreasing map. . . . . . . . . . . . . . . . . . . . . . 486

def.386 Strictly increasing (resp. decreasing) map. . . . . . . . . . . . . . . . . . 486

def.387 Isomorphism between two ordered sets. . . . . . . . . . . . . . . . . . 487

def.388 Good order. . . . . . . . . . . . . . . . . . . . . . . . 487

def.389 Segments. . . . . . . . . . . . . . . . . . . . . . 488

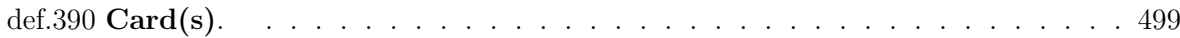

def.391 0, 1. . . . . . . . . . . . . . . . . . . . . . . . 499

def.392 Order on cardinals. . . . . . . . . . . . . . . . . . . . 500

def.393 Sum of cardinals. . . . . . . . . . . . . . . . . . . . . . 506

def.394 $\operatorname{Card}(\mathbf{A})-\operatorname{Card}(\mathbf{B}) \ldots \ldots \ldots \ldots . \ldots . \ldots . \ldots 508$

def.395 a - b. . . . . . . . . . . . . . . . . . . . 509

def.396 Cardinal product. . . . . . . . . . . . . . . . . . 510

def.397 Integers. . . . . . . . . . . . . . . . . . . . . . . . . . . 516

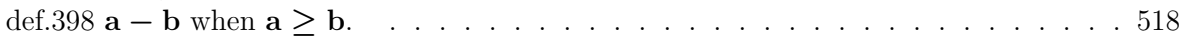

def.399 Numbers. . . . . . . . . . . . . . . . . . . . . . . . . 519

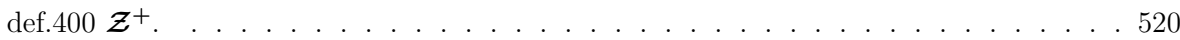

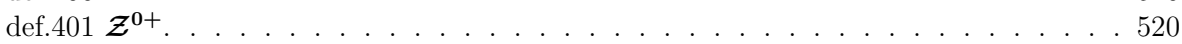

def.402 a/b. . . . . . . . . . . . . . . . . . . . . . . . . . . 522

def.403 Even and odd integer, parity. . . . . . . . . . . . . . . . . . . 522

def.404 Factorial. . . . . . . . . . . . . . . . . . . . . . . . . . . . . . . 528

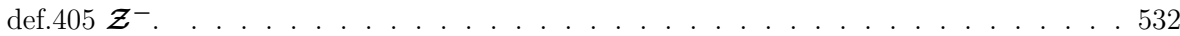

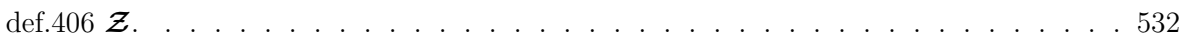

def.407 Absolute value in $\mathcal{Z}$. . . . . . . . . . . . . . . . . . . . . . 532

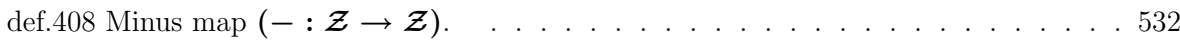

def.409 Order on $\mathcal{Z}$ (definition 406 (p. 532)). . . . . . . . . . . . . . . . 533

def.410 Sum of two elements of $\mathcal{Z}$. . . . . . . . . . . . . . . . . . . . . . 534

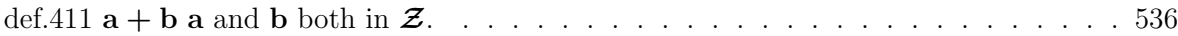


def.412 $\mathbf{a}-\mathbf{b}$ a and $\mathbf{b}$ both in $\mathcal{Z}$. . . . . . . . . . . . . . . . . . . . . 536

def.413 Oriented intervals. . . . . . . . . . . . . . . . . . . . . 537

def.414 Sum of a finite family of elements of $\mathcal{Z}$. . . . . . . . . . . . . . . . . . . . 540

def.415 Product of a finite family of elements of $\mathcal{Z}$. . . . . . . . . . . . . . . . . 543

def.416 Product of two elements of $\mathcal{Z}$. . . . . . . . . . . . . . . . . . . . . . . . 544

def.417 $(-1)^{\mathbf{n}}, \mathbf{n} \in \mathcal{Z}^{0,+}$. . . . . . . . . . . . . . . . . . . . . . . 545

def.418 Rational numbers $\mathcal{Q}$. . . . . . . . . . . . . . . . . . . . . . . . . . 551

def.419 $\mathbf{r}=\mathbf{p} / \mathbf{q} \in \mathcal{Q}$. . . . . . . . . . . . . . . . . . . . . . . . 552

def.420 Notations associated with rational numbers. . . . . . . . . . . . . . . . . 552

def.421 Denominator and numerator of a rational number. . . . . . . . . . . . . . 553

def.422 Sum and product of an integer number of rational numbers. . . . . . . . . . . 554

def.423 Inverse of a rational number. . . . . . . . . . . . . . . . . . . . . . . 554

def.424 Order on $\mathcal{Q}$. . . . . . . . . . . . . . . . . . . . . . . . . . . . . . . . 557

def.425 Euclidean division. . . . . . . . . . . . . . . . . . . . . . . . . 558 


\section{List of conventions}

conv.1 $\left(\partial_{\mu}:[1, n] \rightarrow \mathcal{V}_{\mathrm{k}}\right) \ldots \ldots \ldots \ldots \ldots \ldots$

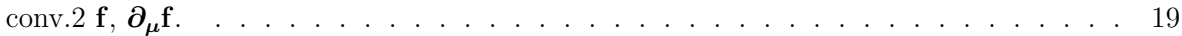

conv.3 $\mathbf{f} \in \mathcal{F}_{\mathrm{k}}, \mathbf{v}(\mathbf{f}) \ldots \ldots \ldots \ldots$

conv.4 $\mathbf{U}_{\mathrm{k}} \rightarrow \mathbf{U}, \mathcal{F}_{\mathrm{k}} \rightarrow \mathcal{F}_{\mathrm{U}}, \mathcal{V}_{\mathrm{k}} \rightarrow \mathcal{V}_{\mathrm{U}} \ldots \ldots \ldots \ldots \ldots$

conv.5 $\left(\partial_{\mu} \mathbf{f}\right) \mathrm{dx}^{\mu}=\partial_{\mu} \mathrm{fdx}^{\mu}$. . . . . . . . . . . . . . . . . . . . . . . . . 35

conv.6 Notation for tensorial product. . . . . . . . . . . . . . . . . . . . . . . 45

conv.7 Notation for a differential on a surface. . . . . . . . . . . . . . . . . . . . . . 66

conv.8 Vectorial space on $\mathcal{C}$. . . . . . . . . . . . . . . . . . . . . . . . . . . . . . . . 87

conv.9 $\mathbf{D}_{\mu}=\mathbf{D}_{\partial_{\mu}}$. . . . . . . . . . . . . . . . . . . . . . . . . . . . . . 91

conv.10 $\mathrm{g}_{00}=1, \mathrm{~g}_{11}=\mathrm{g}_{22}=\mathrm{g}_{33}=-1 \ldots \ldots \ldots$. . . . . . . . 108

conv.11 Conventions in general relativity. . . . . . . . . . . . . . . . . . . . 111

conv.12 Einstein convention. . . . . . . . . . . . . . . . . . . . . 128

conv.13 (f: $\mathbf{A} \rightarrow \mathbf{B}) . \ldots \ldots \ldots . \ldots \ldots \ldots$

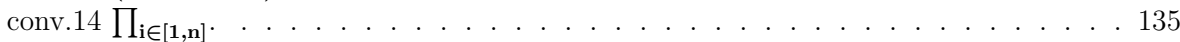

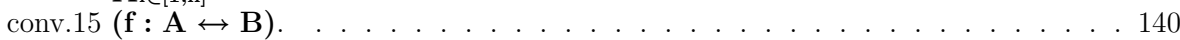

conv.16 Quotation of the inverse of a map. . . . . . . . . . . . . . . . . . . . . 140

conv.17 $\prod_{\mathbf{i} \in \mathbf{I}_{1} \cup \mathbf{I}_{2}}^{\mathrm{e}} \mathbf{x}^{\mathrm{i}} \ldots \ldots \ldots \ldots \ldots \ldots$

conv.18 Sum and product over a finite set of indices. . . . . . . . . . . . . . . . . . 146

conv.19 $\mathbf{V}^{*}$ dual of a module $\mathbf{V}$. . . . . . . . . . . . . . . . . . . . . . . . . . 221

conv.20 $\mathbf{V}_{\mathbf{U}}^{*}$ dual of a module over another module. . . . . . . . . . . . . . . . . 221

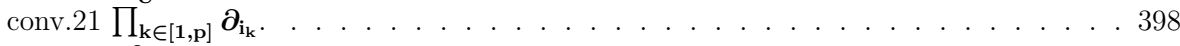

conv.22 $\partial_{\mathrm{k}}^{\mathbf{0}}, \partial_{\mathrm{k}}^{\mathrm{p}} \ldots \ldots \ldots \ldots \ldots \ldots$

conv.23 Substitution in a relation. . . . . . . . . . . . . . . . . . . 433

conv.24 A, B. . . . . . . . . . . . . . . . . . . . . . . . . . . . . . . 434

conv.25 Implicitly true statement. . . . . . . . . . . . . . . . . . . . . . . . . 434

conv.26 Convention with $\mathbf{x}$ when $\exists \mathbf{x R}(\mathbf{x})$ is written as true. . . . . . . . . . . . 441

conv.27 $\mathbf{R}(\mathrm{x})$ stands for $\forall \mathrm{xR}(\mathrm{x})$. . . . . . . . . . . . . . . . . . . . . . . 444

conv.28 $\leq, \geq,<>$. . . . . . . . . . . . . . . . . . . . . 446

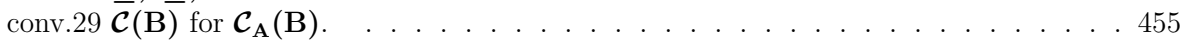

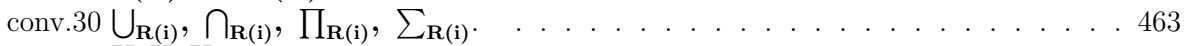

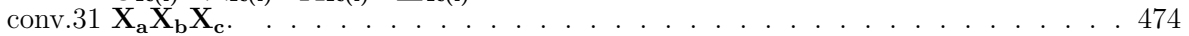

conv.32 $\leq, \geq,<>$. . . . . . . . . . . . . . . . . . . . . . . . . . . . . . 482

conv.33 $\mathbf{a}$ is cardinal. . . . . . . . . . . . . . . . . . . . . . . . . . . . . . . 499

conv.34 $\times_{\mathbf{i} \in \mathbf{I}} \mathbf{a}_{\mathbf{i}}$ and $\prod_{\mathbf{i} \in \mathbf{I}} \mathbf{a}_{\mathbf{i}} \ldots \ldots \ldots \ldots \ldots 14$

conv.35 A, B, C. . . . . . . . . . . . . . . . . . . . . . . 560

conv.36 (ᄏxSy)B. . . . . . . . . . . . . . . . . . . . 560

conv.37 $(\forall \mathbf{x S y})$ B. . . . . . . . . . . . . . . . . . . . . . . . 560

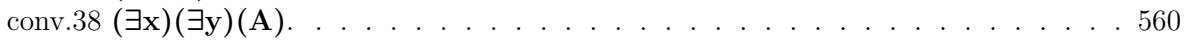

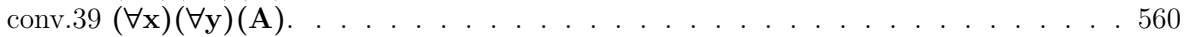

conv.40 $(\forall \mathbf{x})(\exists \mathrm{y})(\mathrm{A}) \ldots \ldots \ldots \ldots 60$

conv.41 $(\exists \mathrm{x})(\forall \mathrm{y})(\mathrm{A}) \ldots \ldots \ldots \ldots 60$

conv.42 More conventions for $\exists$. . . . . . . . . . . . . . . . . . . . . 560 
This page intentionally left blank 


\section{Index}

\author{
A \\ absolute value \\ definition, 181, 532 \\ proposition, 181, 541 \\ text, 118, 361

\section{adjoint} \\ definition, 261, 264, 276, 429 \\ proposition, 30, 265, 429-431 \\ subsection, 429 \\ text, 30, 102, 262, 264, 265, 276, 429-431
}

\section{B}

\section{basic}

chapter, 131, 433

definition, 16, 17

proposition, 23, 35, 54, 72, 109, 220

\section{basis}

definition, 17, 22, 87, 88, 219, 222, 223, $250,267,281$

proposition, 17, 18, 23, 29, 30, 77, 79, 86, 92, 220, 222, 223, 225, 228, 231, 233, 241, 243-246, 248, 250, 252, 256, 257, 262, 264, 267, 270, 274, 275, 305, 324, $333,335,352,357,429-431$

\section{bijection}

definition, 139, 461

proposition, 36, 48, 76, 94, 97, 142-144, 150, 172, 187, 191, 206, 207, 213, 258, 298, 301, 302, 324, 373, 398, 415, 461, 462, 470, 472-475, 489, 498, 506, 511, $518,533,535,545,556$

\section{bijective}

definition, 139, 461

proposition, 68, 143, 149, 150, 187, 401, $507,541,555$

\section{Borel}

definition, 378, 391, 392

proposition, 378

text, 378, 381, 382, 392

\section{boundary}

definition, 67, 157 proposition, $67,74,76,117,158,316,319$, 324

\section{bracket}

definition, 85

proposition, 85, 86

section, 85

text, 85, 99, 109, 261, 434

\section{bundle}

definition, 86, 87, 89-95, 109

proposition, 88-94, 109

section, 86

subsection, 86,96

text, 86-91, 93, 94, 96, 98, 99, 101, 106, 109,110

\section{C}

\section{cardinal}

convention, 499

definition, 500, 506, 510

proposition, 31, 97, 142, 147, 159, 172, $210,211,213,240,241,244,258,275$, $500,504,507,509,510,515,516,518$, $519,525,529,532,549,558$

subsection, 499

\section{characteristic}

definition, 341

proposition, 62 , 342, 347, 349, 352, 355357,360

text, 82, 117, 341-343, 346, 349, 351, 357, $361,366,367,387-389$

close

definition, 154, 179, 181, 286

proposition, 74, 76, 154, 155, 158-160, 165, 178-181, 190, 287-291, 293, 294, 297. 307, 308, 311, 312, 360, 369, 392, 396, 399,401

closure

definition, 155

proposition, 155, 156, 158, 165, 180-182, $287,288,294,314-316,320,343$

\section{commutative}


definition, 206, 215, 218, 219, 221

proposition, 5, 9, 32, 37, 38, 90, 216, 217, 219-223, 225, 226, 228, 230-232, 408, 535

section, 218

text, 5, 8, 9, 17, 38-41, 47, 49, 88, 90, 93, 101, 128, 206, 214-221, 223, 229, 232, 236, 244, 246, 271, 272

\section{compact}

definition, 160, 331, 332, 337, 382

proposition, 160, 162, 164, 167, 180, 184, 189, 190, 290-293, 296, 298, 307, 308, 333, 336, 338-340, 352, 355, 356, 386, 399

section, 331

subsection, 290

\section{complex}

definition, 259, 260, 271, 272, 403

proposition, 260, 272, 273, 404, 411, 412, 415

section, 271

subsection, 272, 403, 411

subsubsection, 412

\section{connected}

definition, 327

proposition, 2, 29, 31, 55, 56, 62, 64, 71, 327-331

section, 326

text, 29, 31, 56, 59, 62, 63, 72, 81, 108, 326-329, 331, 433

\section{connection}

definition, 91, 92, 96, 109, 326

proposition, 91-95, 109, 111, 326

text, 91-96, 98, 99, 101, 109-112, 115, 326, 327,440

\section{continuous}

definition, 165, 183, 184, 382, 383, 392

proposition, 4, 7, 25, 30, 31, 42, 50, 62, 64, 68, 74, 165-169, 182, 184-186, 188193, 195, 196, 198-200, 202, 256, 273, 288, 290-292, 294, 296, 297, 303, 307309, 311, 317, 328, 352, 355-358, 360, 366, 368-370, 373, 382, 392, 396, 399, $401,404,405,418,421,423$

section, 185, 188, 296, 352

subsection, 165

\section{convex}

definition, 67, 71, 277, 280

proposition, 67, 71, 72, 74, 76, 81, 117,
277-281, 285, 288, 312-317, 319, 324, $325,328,350,351,357$

section, 277,312

subsection, 67,74

text, $67,69,71,79,83,105,117,118,126$, 277-280, 312-316, 325, 328, 350, 351, $357,366,369$

\section{coordinate}

definition, 15, 23, 36, 358

proposition, 22, 35, 36, 62, 64, 74, 97, 99, $147,319,357,424$

section, 23

\section{couple}

definition, 448, 449

proposition, 7, 301, 449, 458, 473

text, 1, 3, 5, 26, 33, 88, 114, 159, 276, 393, 448, 449, 458, 461, 473, 493, 529, 530, $550,552,554$

\section{covariant}

definition, 90, 94-96

proposition, 94

subsection, 90

\section{curvature}

definition, 98, 112, 113

proposition, 99

section, 98

text, 99, 101, 112, 113, 116, 123, 127

\section{D}

\section{derivative}

definition, 90, 93-96, 192, 193, 304, 306, 398,403

proposition, 94, 193-196, 198-203, 305, 307309, 311, 356, 366-368, 370, 373, 392,

396, 401, 404, 412, 413, 418, 424, 426

section, 304, 392

subsection, 90, 311

subsubsection, 412

text, 6, 8, 16, 70, 78, 79, 90, 91, 93, 98, 99, 101, 102, 118, 119, 192-197, 200, 202, 269, 304, 306, 310, 311, 358, 369, 372 , 373, 375, 377, 393-395, 397-400, 402-404, 412-414, 426, 427

\section{differential}

convention, 66

definition, 2, 8, 34, 40, 53, 66, 93, 96, 369 proposition, 2, 7, 9, 11, 16, 19, 29, 30, 33$35,38,50,54-56,62,66,67,74,76$, $81,89,109,424$

section, 32, 49, 54, 369 
subsection, 96

text, ix, 2, 3, 16, 19, 34, 35, 37, 39, 40, 44, $45,54,59,62,66,67,71,87,89,90$, 94, 96, 98, 103, 115, 130, 369, 372

\section{dimension}

definition, 1, 2, 67, 71, 109, 240, 250, 256, 280

proposition, 2, 4, 16, 23, 29-31, 35, 47, 50, 54-57, 66, 67, 74, 76, 81, 86, 225, 240, 241, 243-246, 248-251, 253, 256-258, 262, 264-266, 274, 275, 280, 312-314, $316,317,428,430,431$

\section{dual}

convention, 221

definition, 32, 88, 94, 221-223, 244

proposition, 33, 88, 169, 221-223, 228, 232, 245,305

\section{E}

\section{Einstein}

convention, 128

definition, 117, 122

proposition, 233

subsection, 118

subsubsection, 121, 126

text, 109, 117, 118, 122, 123, 126-128, 131, $222,239,323$

\section{electro-weak}

section, 101

text, 101

empty set

definition, 456

proposition, 133, 160, 162, 519

text, 154, 160, 281, 493, 519

\section{equivalence}

definition, 445, 446, 468-470

proposition, 20, 210, 211, 326, 446, 468472,551

subsection, 445, 446, 468

text, 20-23, 211, 234, 327, 445, 446, 468$470,472,500,510,551,552$

\section{Euclidean}

definition, 176, 177, 179, 184, 263, 558

proposition, 30, 176-180, 182-184, 238, 261, $270,312-317,521,558$

section, 176

text, 30, 31, 114, 176, 177, 179, 184, 238, $261,263,265,268,270,285,387,558$

\section{exterior}

section, 49 subsection, 96

G

general relativity

convention, 111

definition, 108, 109

section, 108

text, 105, 108, 111, 117

good order

definition, 487

proposition, 147, 488, 490, 492, 494, 496, 504,532

text, 172, 487, 491-494, 498, 504, 519, 525, 527,530

\section{group}

definition, 205, 206, 211

proposition, 206, 207, 210, 212-215, 219, 248,266

section, 205

subsection, 207, 211

\section{$\mathbf{H}$}

Hausdorff

definition, 159

\section{Heaviside}

definition, 245

proposition, 255

text, 245, 254, 335, 340, 424

\section{Hodge}

definition, 59

text, 59, 67

\section{independent vector}

definition, 239

proposition, 239, 241-244, 251, 280, 285

\section{injective}

definition, 139, 461

proposition, 48, 139, 143, 148-150, 170, $191,213,294,358,360,529$

text, $38,48,75,139,140,148,149,151$ 191, 293, 294, 408, 461, 470, 472, 474, $486,489,502,504,508,513,529,530$, $545,552,559$

\section{inner}

definition, 261, 262, 264, 273, 276, 277, 281

proposition, 262-265, 274, 275, 281, 282, $284,285,312,324,352,357,430,431$ subsection, 259 


\section{integer}

definition, 516, 522, 554

proposition, 147, 160, 164, 171, 172, 187, 202, 207, 212, 246, 278, 297, 306, 307, $312,330,400,516-521,523-525,527-$ $529,535,547$

section, 516

subsection, 521

\section{interior}

definition, 71, 156

proposition, 157, 314-317

\section{interval}

definition, 181, 484, 537

proposition, 147, 181, 190, 191, 195, 279, 288, 316, 349, 392, 393, 399, 415, 484, 488

subsection, 366

text, 181, 189, 190, 192, 279, 316, 317, 320-322, 351, 414, 484, 492

\section{isomorphism}

definition, 166, 487

proposition, 23, 30, 75, 148, 166, 172, 487, $488,495,496,498,532$

text, $1-3,23,75,82,83,146,166,167$, 170, 399, 487, 496-498, 504, 527, 530, 532

\section{K}

\section{Kelvin}

subsection, 74

\section{Lagrangian}

definition, 117

section, 101

text, 101, 102, 104, 105, 109, 117, 118, 122

\section{Lebesgue}

definition, 382, 383, 391, 392

proposition, 386, 389

section, 377

text, $61,171,377,382,383,389$

\section{length}

definition, 274

proposition, 274, 275

\section{Levi-Civita}

definition, 109

proposition, 109, 111

text, 109, 111, 112, 115

\section{limit}

definition, 182, 298, 301, 302, 485 proposition, 172, 174, 175, 182, 183, 189, $287,298-303,311,413,485,559$

subsection, 174

text, ix, 40, 77, 79, 104, 170, 174, 175, 178, 180, 182, 183, 187, 189, 190, 192, 194, 279, 287, 289, 290, 295, 298-303, 312, $317,322,335,349,355,368,372,375$, 394, 398, 403, 407, 409, 419, 449, 485, 522,559

\section{linear}

definition, 40-42, 47, 67, 89, 93, 220, 239, $244,250,256,258,429$

proposition, 5, 37, 38, 42, 46, 47, 89, 91, $92,94,99,112,220,221,224,239-243$. $245,246,251,256-259,265,266,297$. 428-431

section, 45,428

subsection, 244, 250, 429

\section{local}

definition, 17, 36, 61

proposition, 18, 23, 29, 30, 33-36, 55, 56. $62,66,97$

\section{M}

\section{majoring}

definition, 484

proposition, 174, 485, 494, 519

\section{manifold}

chapter, 1,131

definition, 1-3, 8, 13, 17, 23, 25, 32, 40, 61,67

proposition, $2-4,7,9,11,13,16,19,20$, 25-31, 33-35, 38, 47, 50, 53-57, 62, $64,66,67,72,74,76,79,81,86,88$, 89, 109

section, $1,8,25,54,86$

subsection, 67

text, ix, 1-6, 8, 9, 14, 16, 19, 20, 23, 25, 28, $31,34,35,38-41,43-45,53,54,58,59$, 61, 62, 65-67, 71, 85-87, 89-91, 93, 96 98, 101, 105, 108, 109, 111-115, 118, $128,129,218,219,259,312,424$

\section{map}

convention, 140

definition, 15, 16, 53, 89, 90, 97, 131-135, $139,140,145,146,165,182-184,206$, $220,223,225,244,245,302-304,306$ $331,332,341,382,383,392,403,460$ $461,486,532$ 
proposition, 4, 5, 9, 11-13, 16, 22, 25, 26, 29-31, 33-35, 37, 38, 42, 43, 48-50, 53-55, 57, 62, 64, 66-68, 72, 74-76, 85, 95, 132-134, 136, 139-143, 146150, 152, 165-170, 173, 176, 182-191, 198-203, 205-208, 210-221, 224-228, 230, 231, 237, 238, 245-248, 253, 255, 256, 261, 270, 273, 277, 278, 284, 287. 288, 290-292, 294, 296, 297, 301-303, 305, 307-309, 311, 312, 317, 319, 323, $324,328,330,333,335,336,338-340$, $342,345-349,351,352,355-358,360$, 366-370, 382, 400, 401, 403-405, 412, 418, 421, 423, 424, 426, 427, 429, 461, 462, 464-469, 471-481, 483, 486-490, 492, 504-508, 510-515, 527, 529, 532, $533,535,541-548,550,553,555-559$ section, 185, 188, 296, 302, 331, 352 subsection, 131, 165, 311

\section{maximal}

definition, 485

proposition, 494

text, 104, 240, 241, 485, 495

\section{maximum}

proposition, 195, 337

text, 118, 195, 240, 280, 300, 351, 361, $374,383,428$

\section{measure}

definition, 382, 383, 391

proposition, 386, 389

text, 382, 383, 389, 392

\section{metric}

definition, 25, 32, 40, 45, 58, 96, 108, 176, $177,179,184,273,276$

proposition, 25-31, 38, 99, 109, 176-184, $238,247-249,256,261,270,288,312-$ 317,413

section, 25, 176, 181

subsection, 247, 273

subsubsection, 123

minimal

definition, 485

text, 104, 485

\section{minimum}

definition, 176

proposition, 195

text, 195

\section{minoring}

definition, 484 proposition, 174, 485

\section{module}

convention, 221

definition, 87, 88, 218, 219, 221, 223

proposition, 5, 9, 32, 88-92, 99, 219-223, $225,226,228,230-232,415$

section, 218

text, $8,9,17,87-94,96,99-101,218-223$, $226,229,232,236,244,246$

\section{multilinear}

definition, 90, 95, 223, 225, 246

proposition, 44, 224-228, 230, 231, 246, 249

subsection, 246, 247

text, 40-45, 50, 90, 95, 100, 223-225, 228$230,248,249,252,358$

\section{$\mathrm{O}$}

open

definition, 13, 25, 32, 34, 151, 177, 181, $285,286,383$

proposition, 2-4, 7, 9, 11, 13, 19, 20, 25, 29-34, 42, 50, 55, 62, 66, 67, 72, 74, $76,81,86,109,117,151,152,154$, $157,158,177,179,181,183,191,193$, 205, 238, 256, 279, 287-292, 294, 297, 300, 305, 307-309, 311-313, 315-317, $319,320,324,328-331,358,360,373$, $378,382,384,389,403,423,427,428$

\section{operator}

definition, 53, 59, 304

\section{order}

definition, 171, 211, 446, 447, 482, 483, $485,487,500,533,557$

proposition, 23, 37, 147, 171, 305, 370, $373,392,396,399,401,423,447,483$ 490, 494-496, 498, 500, 504, 531, 533, $534,557,558$

subsection, 171, 446, 482, 557

\section{oriented}

definition, 54, 61, 65, 537

proposition, 54-57, 62, 64, 66, 79, 81

subsection, 61

text, 54, 61, 62, 65-67, 83, 105

\section{orthogonal}

definition, 263, 274

proposition, 264, 274, 275, 430, 431

text, 31, 32, 58, 263, 264, 274-276, 314, 430,431 


\section{$\mathbf{P}$}

parallel

definition, 98

section, 98

text, 98, 322-324

\section{parity}

definition, 522

proposition, 523

text, $248,522,545$

\section{partial}

definition, 304, 306, 398

proposition, 16, 305, 307-309, 311, 356, 401

section, 304

text, 16, 118, 119, 304, 306, 311, 358, 375, $398,400,404$

\section{partition}

definition, 471

proposition, 134, 186, 188, 210, 211, 270 , 323, 335, 399, 471, 481, 506, 508, 511, $541,546,556,557$

text, 210, 211, 234, 326, 401, 471

\section{permutation}

definition, 45, 207, 208, 210, 232

proposition, 46, 48, 207-210, 212, 248, 398, 401

text, 45-49, 59, 60, 145, 207-212, 233, 247-249, 252, 254-256, 429

\section{polynomial}

definition, 416, 421

proposition, 31, 416-419, 421-423, 428430

section, 416

text, 30, 31, 416-419, 421, 422, 424, 425, 429-431

\section{product}

convention, 45, 146

definition, 8, 40-42, 45, 95, 134-136, 145, 146, 153, 211, 230, 250, 261, 262, 264, 273, 276, 277, 304, 398, 459, 473, 510, $543,544,554$

proposition, 9, 42, 46-48, 50, 52, 56, 57, $147-149,154,162,164,166,185,186$, 206-209, 221, 230, 243, 251, 256, 257, 262-266, 274, 275, 286, 296, 306, 307, 312, 352, 356, 416, 430, 431, 472, 486, 523,549

subsection, 186-188, 250, 259, 458, 473

subsubsection, 555-557

\section{$\mathbf{R}$}

\section{rational}

definition, 551-554

proposition, 171, 172, 551, 554, 555, 558

section, 551

subsection, 552, 553

real

definition, 170, 181, 332

proposition, 30, 171, 172, 190, 277, 278, $280,312,315-317,366,423,429$

section, 170

subsection, 171

\section{restricted}

definition, 4, 32, 34

proposition, 3, 4, 13, 19, 26, 30, 33-35, 54, 86, 109, 139, 143, 166, 169, 183, 246, 305

text, $3,4,13,32,34,43,48,51,56,59,91$, 106, 109, 129, 143-145, 176, 183, 186. $202,219,224,246,259,270,488$

\section{$\mathrm{S}$}

\section{segment}

definition, 488

proposition, 171, 172, 193, 196, 488-490, $492,495,496,498,525,559$

text, 170-172, 174, 488-490, 492-499, 504, $525,527,532,559$

\section{separated}

definition, 159

proposition, 159, 160, 162, 179, 189, 238, 256

text, 159-162, 180

\section{signature}

definition, 32, 208, 276

proposition, 209, 210, 248

text, 32, 46, 47, 59, 60, 102, 108, 109, 116 . 209, 233, 247, 248, 252, 254, 256, 276, 429

\section{smooth map}

definition, 3-5, 8, 306

proposition, 3, 4, 6, 7, 9, 13, 35, 57, 66, 427, 428

section, 3,424

text, $3-5,8,9,14,16,19,20,34,58,87-$ 91, 93, 101, 109, 128, 218, 424

\section{Stokes}

subsection, 74

text, 120

subset 
definition, 13, 32, 132, 133, 135, 151, 154$157,176,177,184,211,240,277,280$, $327,341,342,483,485$

proposition, 2, 11, 29, 31, 34, 48, 54, 62, $64,67,72,74,76,97,147,151-160$, 162, 165-169, 177-180, 184, 189, 240, 244, 248, 275, 278, 280, 281, 288-291, 293, 294, 296, 303, 312, 314-317, 319, 325, 328-331, 342, 343, 345, 348, 350, 355-357, 377, 415, 457, 483, 485, 488, $490,494,498,503,504,525,534,551$ section, 277,312

subsection, 174, 451, 454

\section{surjective}

definition, 139, 461

proposition, 139, 140, 142, 149, 305, 323, $465,467,471$

text, 37, 38, 48, 75, 86, 139, 140, 142, 149, 150, 191, 323, 409, 461, 470, 472, 474, $489,506,508,513,529,531,545,552$

\section{$\mathbf{T}$}

tangent space

definition, 20-22, 109

proposition, 20, 22, 23, 27, 28, 30, 31, 109 section, 19

text, 20, 21, 28, 31, 32, 98, 109, 110, 114

\section{tensorial field}

section, 40

\section{topology}

definition, 151, 153, 159, 184, 286

proposition, 74, 151, 152, 154, 155, 162, 165, 166, 177, 181, 238, 273, 286, 288, $293,294,312,313,315-317,331$

section, 181, 285, 312

subsection, 151

text, 3, 66, 75, 87, 114, 151, 153, 155, 162, $178,183,184,189,268,286,290,293$, $294,314,327,329,382,403,561$

\section{total order}

definition, 483

proposition, 171, 488, 500, 531, 532

text, 146, 504

\section{transformation}

definition, 89, 93, 220, 250, 256, 264, 265, $276,277,429$

proposition, 30, 89, 91, 92, 94, 250, 251, 256-258, 265, 266, 297, 428-431

section, 428

subsection, 250, 429

\section{transport}

definition, 98

section, 98

text, 98

\section{transposition}

definition, 208, 252

proposition, 208, 209, 247

text, 47, 62, 88, 208, 209, 234, 248, 254, 255

\section{$\mathrm{U}$}

\section{uniformly}

definition, 184, 302, 337

proposition, 184, 303, 311, 338, 351, 352

text, 184, 189, 291, 293, 302, 303, 312, $337,356,372,393,397,400$

\section{unitary}

definition, 265, 277

proposition, 265

text, 102, 103, 106, 110, 265, 271, 277, 360

\section{vector field}

definition, $8,13,14,16,17,32,40-42,85$, 109

proposition, 8, 9, 11-13, 18, 19, 23, 27, 30, $32,33,35,42,47,54,56,66,85,86$, 99, 109

section, 8,45

subsection, 8,9

text, 8, 9, 13, 14, 17-20, 25, 26, 28, 32-34, $36,37,40,41,43,45,47,54,56,58$, $59,85,90,91,93,94,96,98,101,102$, 108-110, 128-130

\section{vectorial space}

convention, 87

definition, 237, 239, 240, 242, 244, 246, 256, 276, 405

proposition, 5, 238-247, 249-251, 253, 256258, 261, 262, 264-266, 270, 274, 275, 277-280, 282, 284, 301, 305, 312-317, $406,408,431$

text, 5, 8, 16, 58, 87-90, 93, 96, 98, 100, 101, 237, 239, 240, 242, 244-246, 248, 250-252, 256-258, 261-265, 271, 272, $276,277,280,281,300,325,331,405$ 407, 411, 428, 429, 431

\section{volume}

definition, 54, 58, 342 
proposition, 54-57, 62-64, 79, 342, 343, $348,349,351,352,356,357,360,386$, 389

section, 54, 341

subsection, 54

text, 54, 56, 58, 59, 61, 62, 65, 67, 82, 83, $342,343,351,352,358,361,363,366$, $377,388-390$

W

wedge product

definition, 47, 97

proposition, 47, 54

section, 45

text, 47 